\title{
Un'insolita astenia: un caso di cardiomiopatia dilatativa in un giovane con dissezione coronarica spontanea
}

\author{
A strange weakness: a case-report of dilated cardiomyopathy \\ in a young patient with spontaneous coronary dissection
}

\section{Francesco Bartolomucci $^{a, *}$, Francesco Cipriani ${ }^{a}$, Giovanni Valente $^{a}$, Serafino Curci $^{\text {b }}$, Giovanni Deluca ${ }^{c}$}

\author{
a UOC Cardiologia-UTIC, Ospedale L. Bonomo, Andria, Barletta \\ ${ }^{\mathrm{b}}$ Divisione di Medicina Interna, Casa di Cura Sollievo della Sofferenza, San Giovanni Rotondo, Foggia \\ ${ }^{\mathrm{C}}$ UOC Cardiologia, Ospedale Civile, Bisceglie, Barletta
}

Ricevuto il 13 settembre 2011; accettato il 17 novembre 2011

disponibile online il 17 marzo 2012

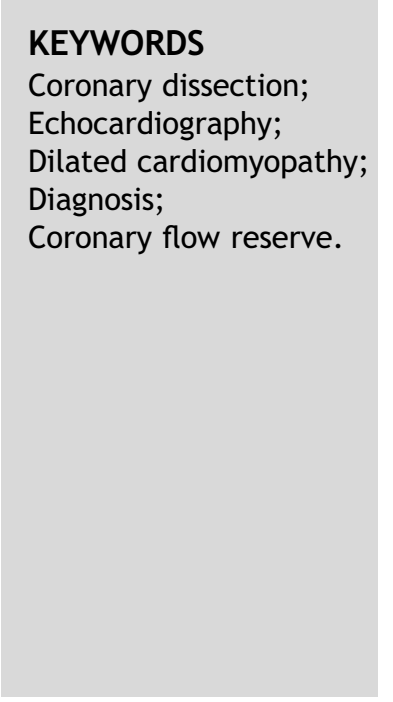

\begin{abstract}
Summary
Introduction: Atherosclerosis is the most common cause of coronary affections, but coronary dissection is certainly more insidious. This is the separation of the media apart from the other layers of the vessel wall, with or without intimal tear. Spontaneous coronary dissection is a rare event, especially in young men: only 150 cases were reported till 1986 and over 300 till date. The main consequence is an acute coronary occlusion with myocardial infarction and sudden death. Case report: We report the case of a 28 -year-old man, presented with a mild symptomatic spontaneous coronary dissection, consisting of a strange weakness, remained undetected until fortuitous medical analyses suggested the opportunity of further investigations. A clinical examination and an EKG showed the opportunity to perform a bidimensional echocardiogram at first, a low-dose dobutamine stress echocardiogram, then a regional myocardial perfusion and a non-invasive coronary flow reserve assessment. The patient has been properly and quickly screened and is now enlisted for cardiac transplantation.

Conclusions: In such cases the immediate identification and treatment of the affection can be crucial. In our experience the basic and advanced echocardiography allowed a sooner diagnosis than the usual proceeding based on coronarographic examination.

(c) 2012 Elsevier Srl. All rights reserved.
\end{abstract}

\footnotetext{
* Corrispondenza: UOC Cardiologia-UTIC, Ospedale L. Bonomo, via San Candido 75 - 70100 Andria, Barletta.

E-mail: francescobartolomucci@virgilio.it (F. Bartolomucci).
} 


\section{Introduzione}

La dissezione coronarica è per definizione una separazione della tunica media dagli altri strati della parete vascolare, con o senza breccia di lacerazione intimale, causata da una lesione emorragica capace di spingere lo strato intimale verso il centro del lume, ostruendolo [1]. La dissezione coronarica può essere primitiva (spontanea) o secondaria (a traumi toracici, postinfiammatoria o iatrogena) [2]. La dissezione spontanea è un'eventualità estremamente rara, capace di causare un'ostruzione acuta del lume coronarico, conseguendone solitamente infarto miocardico acuto e morte improvvisa [3,4]. Riportiamo il caso di un giovane paziente ventottenne giunto alla nostra attenzione con una dissezione coronarica paucisintomatica, rimasta misconosciuta finché controlli medici occasionali non hanno suggerito un approfondimento diagnostico.

\section{Caso clinico}

Paziente maschio di 28 anni, ex fumatore, con familiarità per cardiopatia ischemica e ipercolesterolemia, normopeso e senza habitus marfanoide, ha iniziato ad avvertire astenia nello svolgimento delle proprie attività quotidiane; in occasione di un periodico controllo eseguito presso la Medicina del Lavoro, si è sottoposto a un elettrocardiogramma (ECG) che ha evidenziato segni di pregressa necrosi anteriore. È così giunto alla nostra osservazione.

L'anamnesi cardiologica remota era muta fino a febbraio 2006, quando aveva presentato dolore toracico irradiato al braccio sinistro per cui si era recato in Pronto Soccorso. All'ECG non erano evidenti segni di ischemia miocardica e il dosaggio enzimatico miocardio-specifico risultava normale. Ad agosto 2006 si era manifestato un nuovo dolore toracico, per cui il paziente si era recato in Pronto Soccorso; sottoposto a esame elettrocardiografico risultato privo di segni di ischemia e veniva perciò trattato con antinfiammatori.

Al momento del ricovero il paziente, che non assumeva alcuna terapia, riferiva solo lieve dispnea da sforzo da qualche giorno (Classe NYHA II). In anamnesi risultavano del tutto assenti i principali fattori di rischio cardiovascolare e quelli associabili a dissezione spontanea (abuso di sostanze, surmenage fisico). La pressione arteriosa era di $115 / 70 \mathrm{mmHg}$ e l'esame obiettivo cardiopolmonare risultava nella norma. L'ECG rilevava un ritmo sinusale a $90 \mathrm{bpm}$ con onde $\mathrm{Q}$ in sede inferiore e scarsa progressione dell'onda $R$ da $V_{1}$ a $V_{5}$. Il radiogramma toracico in due proiezioni mostrava un'ombra cardiaca di dimensioni aumentate, senza segni di versamento in atto, né lesioni polmonari focali. Le analisi ematochimiche erano nella norma. Il paziente, quindi, veniva sottoposto a esame ecocardiografico bidimensionale, che evidenziava una cavità ventricolare sinistra notevolmente dilatata (diametro telediastolico $76 \mathrm{~mm}$; diametro telesistolico $63 \mathrm{~mm}$; volume telediastolico $350 \mathrm{~mL}$; volume telesistolico $270 \mathrm{~mL}$ ), con discinesia del setto interventricolare e dell'apice che apparivano, inoltre, iperecogeni e assottigliati (spessore telediastolico $5 \mathrm{~mm}$ ) e con ipocinesia dei restanti segmenti miocardici, funzione sistolica severamente ridotta (frazione di eiezione $20 \%$ ) e una disfunzione diastolica di $1^{\circ}$ grado (rapporto $E / A=0,69 ; E / E ' 12,5$ ). L'atrio sinistro risultava lievemente dilatato (diametro antero-posteriore $44 \mathrm{~mm}$; volume atriale sinistro $25 \mathrm{~mm}^{3}$ ). Sezione destra nei limiti per dimensioni e normale funzione sistolica del ventricolo destro: TAPSE (Tricuspid Annular Plane Systolic Excursion) $=19 \mathrm{~mm}$. Si riscontravano un'insufficienza mitralica di $1^{\circ}$ grado e un'insufficienza tricuspidalica lieve con pressione sistolica polmonare stimata in $29 \mathrm{mmHg}$. Il paziente veniva sottoposto a eco-stress con dobutamina a basse dosi $(5,10,20 \mu \mathrm{g} / \mathrm{kg} / \mathrm{min})$ e studio della perfusione miocardica con esafluoruro di zolfo sotto forma di microbolle (fig. 1) per la ricerca di miocardio vitale. Durante il test, il paziente rimaneva asintomatico, l'ECG mostrava un lieve sopraslivellamento del tratto ST in $\mathrm{V}_{1}-\mathrm{V}_{6}$ al massimo carico di dobutamina $(20 \mu \mathrm{g})$. Il test mostrava assenza di vitalità contrattile (Wall Motion Score Index pari a 32) e microcircolatoria nel territorio di pertinenza dell'arteria coronarica discendente anteriore.

Successivamente, il paziente è stato sottoposto a studio non invasivo della riserva coronarica sull'arteria interventricolare anteriore mediante ecocardiografia transtoracica con infusione di adenosina (0,14 mg/kg per 90 secondi) (fig. 2). La quantificazione della riserva di flusso coronarico mediante ecocardiografia Doppler transtoracica è un'analisi di recente introduzione per valutare l'entità della malattia ateromasica e la validità della microcircolazione miocardica: la si ricava dal rapporto tra le velocità di flusso coronarico in condizioni di iperemia e basali. Il valore limite per la definizione di normalità è 2 . Si rilevava in questo modo una riserva coronarica pari a 1,6, espressione di importante riduzione della riserva coronarica. Il successivo cateterismo cardiaco condotto per via radiale destra con introduttore da $5 \mathrm{~F}$ (fig. 3) mostrava un'arteria discendente anteriore subocclusa all'ostio con lunga dissezione fino al tratto medio e flussi con punteggio al Thrombolysis in Myocardial Infarction Index pari a 1 a valle, riabilitata da arteria coronarica destra indenne (Rentrop 1-2); l'arteria circonflessa risultava indenne.

L'esame elettrocardiografico delle 24 ore secondo Holter condotto in seguito mostrava frequenti extrasistoli ventricolari, monofocali, talora a coppia, con alcuni brevi run di tachicardia ventricolare non sostenuta. In base ai risultati degli esami condotti e alla luce dei risultati del Multicenter

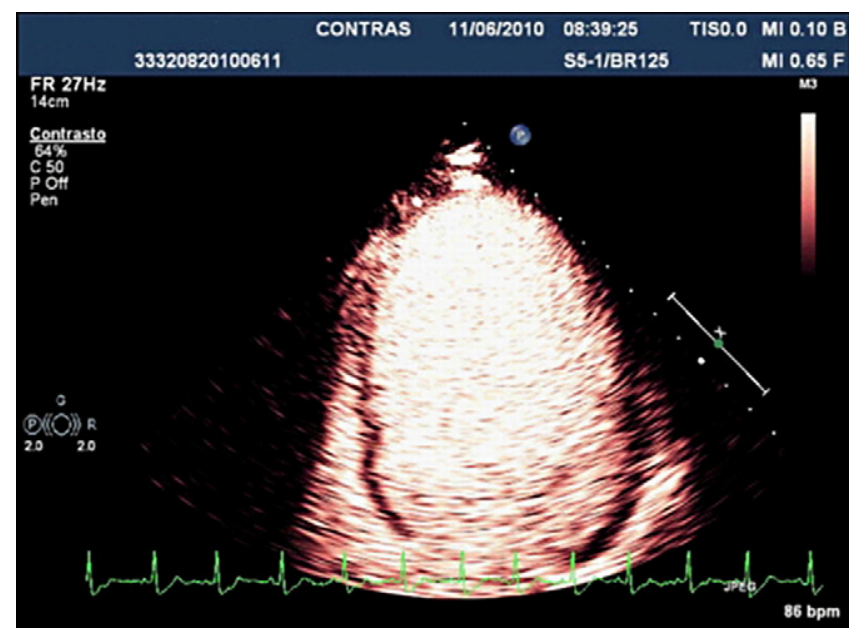

Figura 1 Studio ecocontrastografico con esafluoruro di zolfo sotto forma di microbolle. 


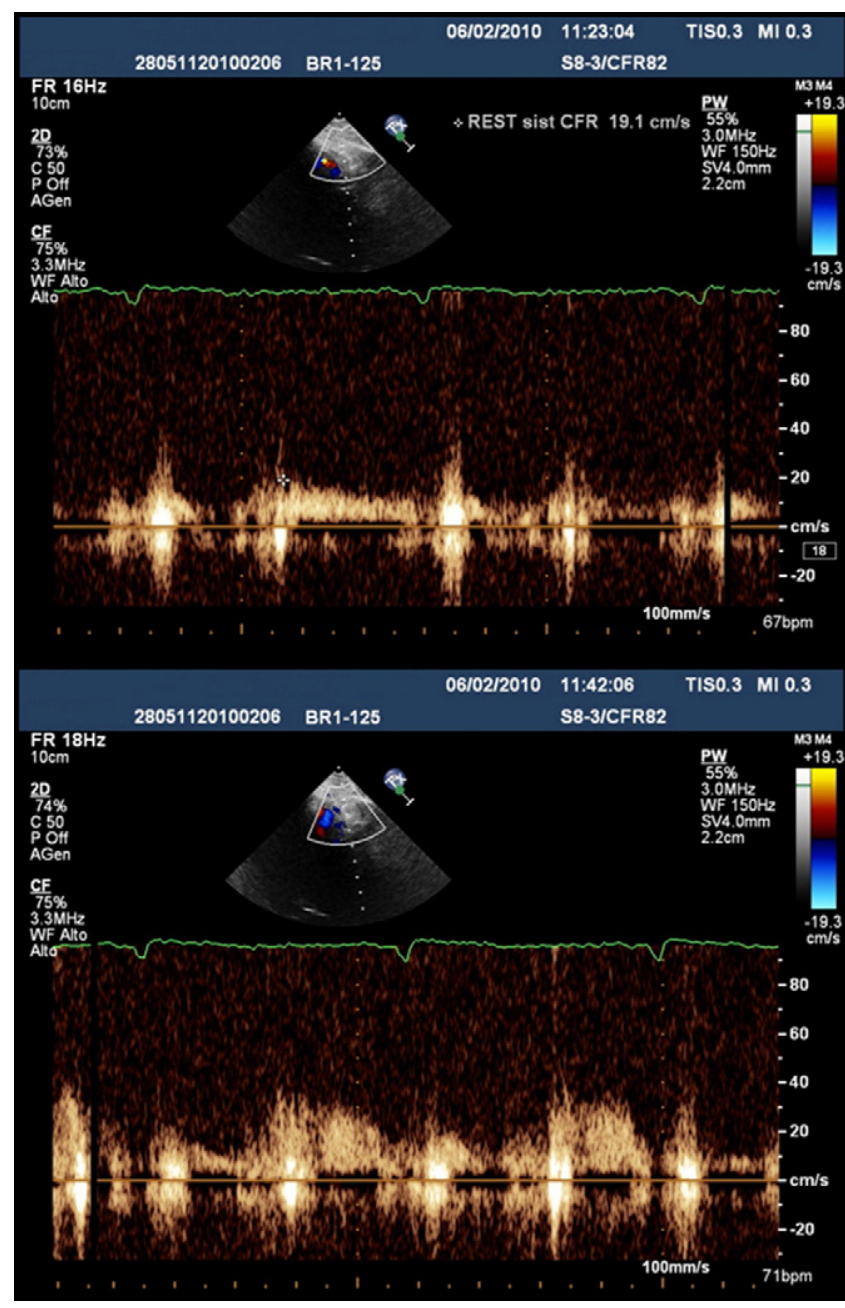

Figura 2 Studio non invasivo della riserva coronarica su arteria interventricolare anteriore distale: basale e postiperemia.

Autonomic Defibrillator Implantation Trial II (MADIT II), si è deciso, pertanto, di procedere all'impianto di defibrillatore cardiaco (Implantable Cardioverter-Defibrillator, ICD) bicamerale ad alta energia di shock. Il paziente è stato dimesso in buone condizioni generali dopo un decorso postimpianto privo di complicanze e con diagnosi di cardiomiopatia dilatativa postischemica. Il mese successivo è stato sottoposto a ulteriori esami riguardanti l'assetto autoanticorpale e i polimorfismi dei geni con particolare riguardo per il lupus eritematoso sistemico e altre collagenopatie. Il paziente non presentava criteri diagnostici riferibili a condizioni di ipercoagulabilità e a sindrome di Marfan secondo la nosologia di Ghent. Dall'anamnesi familiare non emergevano dati suggestivi per sindrome di Marfan in altri familiari. La dissecazione coronarica sembrava essere correlata a un coinvolgimento aterosclerotico delle coronarie, ma per la giovane età del paziente sono stati analizzati i geni correlati a patologia aneurismatica ottenendo riscontri negativi.

Prima della dimissione il paziente è stato sottoposto a risonanza magnetica nucleare (RMN) per lo studio della vitalità miocardica, con esito negativo per vitalità miocardica nel territorio dell'arteria interventricolare anteriore, e a test cardiopolmonare condotto durante copertura farmacologica con protocollo a rampa I interrotto a $50 \mathrm{~W}$ con

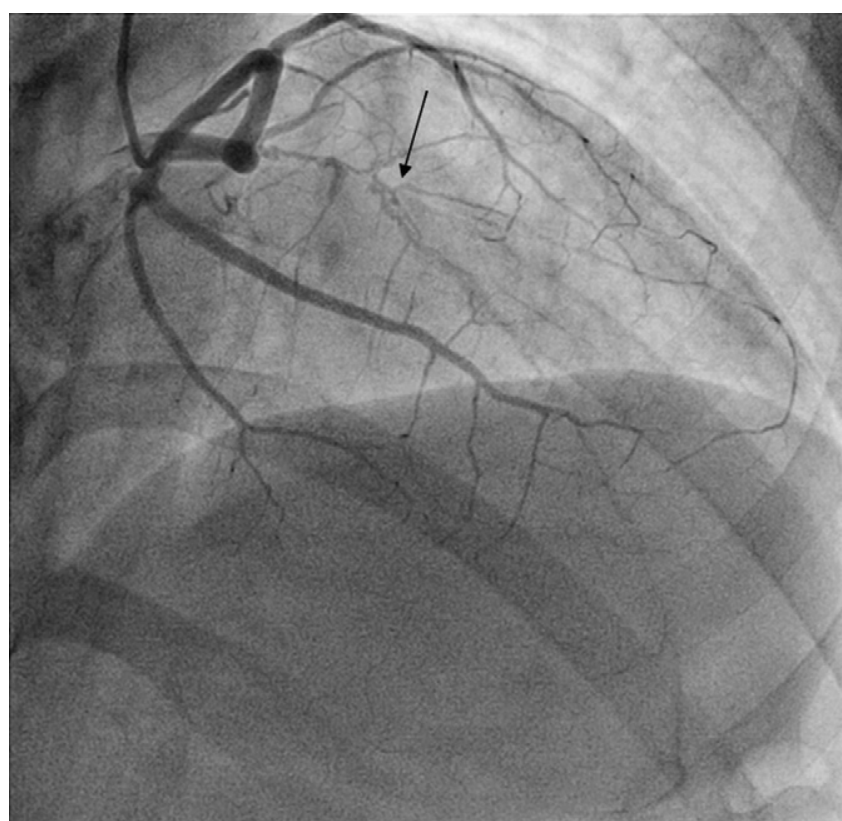

Figura 3 Studio coronarografico: la freccia indica il ramo interventricolare anteriore dell'arteria coronaria sinistra, con un'immagine di doppio lume tipica di una dissezione.

paziente sintomatico per dispnea ingravescente. L'ECG basale e al termine del test mostrava ritmo sinusale e assenza di aritmie significative, non segni di ridotta riserva coronarica, normale risposta pressoria. Il test massimale per RQ raggiunto $(0,98)$ era indicativo di ridotta capacità funzionale $\left(\mathrm{VO}_{2 \text { Max }}\right.$ pari a $\left.14,2 \mathrm{~mL} / \mathrm{kg} / \mathrm{min}\right)$ con segni di limitazione cardiogenica e/o decondizionamento, assenza di segni di limitazione vascolare polmonare e di limitazione ventilatoria $(30,3 \mathrm{~L} / \min \max )$. La saturazione di $\mathrm{O}_{2}$ al picco del $98 \%$.

Non è stato ritenuto utile, ai fini prognostici e del percorso terapeutico, verificare con tomografia computerizzata e coronarografia l'eventuale risoluzione del quadro di dissezione coronarica, poiché questa era stata sicuramente identificata come causa del quadro ecocardiografico e, seppur transitoria, aveva determinato un danno miocardico irreversibile come documentato dall'assenza di vitalità all'ecostress con dobutamina e alla RMN e dal deficit di perfusione all'esame ecocontrastografico.

Sulla base degli esami ecocardiografici di base e da stress, delle indagini coronarografiche e degli esami genetici, il paziente è stato affidato al Centro per lo Studio e la Cura dello Scompenso Cardiaco di Bergamo per una stretta osservazione clinica e strumentale, in vista di un eventuale futuro trapianto cardiaco.

È stato ottenuto il consenso informato da parte del paziente.

\section{Discussione}

La dissezione coronarica spontanea è un evento raro e, sin dalla sua prima descrizione fornita da Pretty nel 1931 [5], ne sono noti pochi casi, in maggior parte (70-75\%) diagnosticati a posteriori con l'esame autoptico [6]. Circa i due terzi dei casi riportati in letteratura riguardano il sesso femminile in stato gravidico [7] o durante trattamento con anticoncezionali 
orali [8]. Ulteriori fattori correlati all'insorgenza di una dissezione coronarica spontanea sono l'uso di cocaina [3], intensi stress emotivi [9] oppure uno strenuo stress fisico, come rilevato nel $28 \%$ del campione esaminato da Hering et al. [10]. La patogenesi non è chiara e in alcuni casi potrebbero essere coinvolte malformazioni congenite del collagene [11] e in modo particolare la panarterite nodosa [12], ipotesi che si è ritenuto opportuno testare anche nel nostro caso, pur senza ottenere riscontro. Solo in pochissimi casi è riconoscibile una breccia intimale che innesca il processo di dissecazione, con l'ipotesi che l'ematoma possa essere generato da un sanguinamento di vasa vasorum [13] e che, quindi, la breccia, quando riscontrabile, sia un punto di fuoriuscita di sangue dallo spessore della parete vascolare [14], anziché un punto d'ingresso. La formazione di ateromi può produrre una dissecazione spontanea come risultato del rilascio di proteasi da parte degli eosinofili che vi si accumulano [15].

La comprensione di quest'affezione è limitata dalla scarsità di case report in letteratura e, per questo motivo, non esistono ancora chiare indicazioni circa prognosi e trattamento. A tutt'oggi appare chiaro solamente come la prognosi a lungo termine sia buona, a condizione di superare senza complicazioni la fase acuta [16]. La gravità di tale condizione e la severità della prognosi quoad vitam e quoad valetudinem impongono un alto indice di sospetto in caso di sintomi insorgenti bruscamente e con andamento "a poussée". La diagnostica ecocardiografica, sia di base sia avanzata, ha permesso in questo caso di porre il sospetto di una cardiomiopatia dilatativa postischemica. Il test di riserva coronarica condotto sull'arteria discendente anteriore ha mostrato la sua utilità clinica in termini diagnostici evidenziando come la grave disfunzione contrattile osservata in sede setto-apicale possa essere stata originata da due meccanismi eziopatogenetici concomitanti: stenosi fissa dell'arteria discendente anteriore e ampia distruzione di unità microcircolatorie. Il test ha confermato l'ipotesi di una cardiomiopatia dilatativa postischemica, e non dilatativa primitiva, sulla base soprattutto dell'assottigliamento della parete miocardica e della sua iperecogenicità.

La diagnosi in vivo è quasi sempre affidata a indagini coronarografiche: si nota il riempimento del falso lume da parte del mezzo di contrasto, con una sua ritardata clearance [13]. In assenza di una breccia intimale, data la minima o nulla comunicazione con l'ematoma, solo un'indagine mirata della parete del vaso può chiarire la diagnosi, usando metodiche invasive come l'Intra-Vascular Ultrasound (IVUS) [2,17] o l'Optical Coherence Tomography (OCT) [18]. Nel caso specifico non è stato possibile applicare tali metodiche a causa della subocclusione dell'arteria coronaria.

Le opzioni per il trattamento di questi pazienti sono varie, in rapporto alla situazione clinica e alla localizzazione ed estensione della lesione. I pazienti asintomatici con buon compenso emodinamico possono giovarsi del semplice trattamento medico $[19,20]$ con nitrati, calcio-antagonisti e beta-bloccanti [21]. Nel nostro caso si è seguita una strategia analoga e con impianto di un ICD per la presenza di turbe del ritmo riscontrate con l'esame Holter.

Altre opzioni terapeutiche sono i trattamenti con agenti trombolitici [22] e antiaggreganti [23], talvolta usati con successo, sebbene espongano al rischio che la dissoluzione del trombo possa riaprire il falso lume al flusso sanguigno e far progredire la dissecazione intimale [24]. La rivascolarizzazione è indicata solo in caso di diagnosi tempestiva [13] e può essere effettuata per via percutanea o per via chirurgica $[25,26]$, pur permanendo le difficoltà nel localizzare il vero lume e stabilire il punto esatto per il pontaggio del bypass.

\section{Conclusioni}

Il caso in esame mette in luce la potenziale complessità del processo di diagnosi differenziale nell'indagine di un sintomo comune e del tutto aspecifico come l'astenia. La notevole rarità di un'affezione come la dissezione coronarica spontanea, per giunta in un paziente non certo identificabile come "a rischio", non dovrebbe permettere di escluderla a priori dalle ipotesi da vagliare nel corso degli accertamenti. In casi come questo, ogni ausilio utile a individuare l'origine del problema e a trattarlo in modo tempestivo e adeguato deve ritenersi di fondamentale importanza. La diagnostica ecocardiografica, sia di base sia avanzata, può permettere di porre diagnosi differenziale tra una forma di cardiomiopatia dilatativa primitiva e una forma secondaria postischemica, in anticipo rispetto a quanto solitamente consentito dalla coronarografia e con conseguente stratificazione prognostica del caso.

\section{Conflitto di interessi}

Gli autori dichiarano di non aver nessun conflitto di interessi.

\section{Bibliografia}

[1] Wayne A, Schlant RC. Arteries and veins. In: Fuster V, O'Rourke R, Walsh R, Poole-Wilson P, King S, Roberts R, et al., editors. Hurst's The Heart.. New York, NY: McGraw-Hill; 1998. p. 1366.

[2] Morocutti G, Spedicato L, Vendrametto F, Bernardi G. Intravascular echocardiography (ICUS) diagnosis of post-traumatic coronary dissection involving the common trunk. A case report and review of the literature. G Ital Cardiol 1999;29(9):1034-7.

[3] Kamran M, Guptan A, Bogal M. Spontaneous coronary artery dissection: case series and review. J Invasive Cardiol 2008; 20(10):553-9.

[4] Kamineni R, Sadhu A, Alpert JS. Spontaneous coronary artery dissection: report of two cases and a 50-year review of the literature. Cardiol Rev 2002;10(5):279-84.

[5] Pretty HC. Dissecting aneurysm of coronary artery in a woman aged 42: rupture. Br Med J 1931;1:667.

[6] Jorgensen MB, Aharonian V, Mansukhani P, Mahrer PR. Spontaneous coronary dissection: a cluster of cases with this rare finding. Am Heart J 1994;127(5):1382-7.

[7] DeMaio Jr SJ, Kinsella SH, Silverman ME. Clinical course and long-term prognosis of spontaneous coronary artery dissection. Am J Cardiol 1989;64(8):471-4.

[8] Azam MN, Roberts DH, Logan WF. Spontaneous coronary artery dissection associated with oral contraceptive use. Int J Cardiol 1995;48(2):195-8.

[9] Hendiri T, Bonvini RF, Martin W, Doriot PA, Camenzind E. Acute myocardial infarction due to spontaneous coronary artery dissection. Arch Mal Coeur Vaiss 2005;98(10):974-8.

[10] Hering D, Piper C, Hohmann C, Schultheiss HP, Horstkotte D. Prospective study of the incidence, pathogenesis and therapy of spontaneous, by coronary angiography diagnosed coronary artery dissection. Z Kardiol 1998;87(12):961-70. 
[11] Thistlethwaite PA, Tarazi RY, Giordano FJ, Jamieson SW. Surgical management of spontaneous left main coronary artery dissection. Ann Thorac Surg 1998;66(1):258-60.

[12] Chu KH, Menapace FJ, Blankenship JC, Hausch R, Harrington T. Polyarteritis nodosa presenting as acute myocardial infarction with coronary dissection. Cathet Cardiovasc Diagn 1998;44(3): $320-4$.

[13] Dhawan R, Singh G, Fesniak H. Spontaneous coronary artery dissection: the clinical spectrum. Angiology 2002;53(1):89-93.

[14] Mulvany NJ, Ranson DL, Pilbeam MC. Isolated dissection of the coronary artery: a postmortem study of seven cases. Pathology 2001;33(3):307-11.

[15] Robinowitz M, Virmani R, McAllister Jr HA. Spontaneous coronary artery dissection and eosinophilic inflammation: a cause and effect relationship? Am J Med 1982;72(6):923-8.

[16] Mortensen KH, Thuesen L, Kristensen IB, Christiansen EH. Spontaneous coronary artery dissection: a Western Denmark Heart Registry study. Catheter Cardiovasc Interv 2009;74(5):710-7.

[17] Maehara A, Mintz GS, Castagna MT, Pichard AD, Satler LF, Waksman R, et al. Intravascular ultrasound assessment of spontaneous coronary artery dissection. Am J Cardiol 2002; 89(4):466-8.

[18] Gutiérrez García H, del Amo Hernández E, Joseph Arnold R, San Román JA. Coronary artery dissection assessed by optical coherence tomography. Rev Esp Cardiol 2009;62(5):587-8.
[19] Sarmento-Leite R, Machado PR, Garcia SL. Spontaneous coronary artery dissection: stent it or wait for healing? Heart 2003;89(2):164.

[20] Evangelou D, Letsas KP, Korantzopoulos P, Antonellis I, Sioras E, Kardaras F. Spontaneous coronary artery dissection associated with oral contraceptive use: a case report and review of the literature. Int J Cardiol 2006;112(3):380-2.

[21] Vale PR, Baron DW. Coronary artery stenting for spontaneous coronary artery dissection: a case report and review of the literature. Cathet Cardiovasc Diagn 1998;45(3):280-6.

[22] Behnam R, Tillinghast $S$. Thrombolytic therapy in spontaneous coronary artery dissection. Clin Cardiol 1991;14(7):611-4.

[23] Cheung S, Mithani V, Watson RM. Healing of spontaneous coronary dissection in the context of glycoprotein IIB/IIIA inhibitor therapy: a case report. Catheter Cardiovasc Interv 2000;51(1): 95-100.

[24] Buys EM, Suttorp MJ, Morshuis WJ, Plokker HW. Extension of a spontaneous coronary artery dissection due to thrombolytic therapy. Cathet Cardiovasc Diagn 1994;33(2):157-60.

[25] Bonacchi M, Prifti E, Giunti G, Frati G, Leacche M, Brancaccio G, et al. Emergency management of spontaneous coronary artery dissection. J Cardiovasc Surg 2002;43(2):189-93.

[26] Hong MK, Satler LF, Mintz GS, Wong SC, Kent KM, Pichard AD, et al. Treatment of spontaneous coronary artery dissection with intracoronary stenting. Am Heart J 1996;132(1 Pt 1):200-2. 